\title{
Special issue on sporotrichosis: challenges to deal with the new emerging pathogenic species
}

\author{
Leila M. Lopes-Bezerra ${ }^{1}$. Sandro Rogério de Almeida ${ }^{2}$
}

Received: 15 December 2020 / Accepted: 28 January 2021 / Published online: 18 February 2021

(C) Sociedade Brasileira de Microbiologia 2021

This special issue of the Brazilian Journal of Microbiology is dedicated to human and animal sporotrichosis. This is a national and international effort of the ISHAM Working Group on Sporothrix and Sporotrichosis (ISHAM/WG18) to stimulate and disseminate the knowledge in one of the most important emerging mycoses of the last decade, sporotrichosis. Since the creation of the ISHAM Working Group on Sporothrix and Sporotrichosis by Dr. Leila M Lopes Bezerra (Brazil) and Dr. Hector Mora-Montes (Mexico), in 2011, the ISHAM/WG18 have been organizing international scientific meetings to promote an interdisciplinary and translational approach to put together the basic and the clinical research on this subject. It is our pleasure to share with the Medical Mycology community this special issue of BJM and also, the information collected for this Editorial about the number and quality of publications on sporotrichosis.

A brief search on PubMed using a combination of timelines (1975 to 2020) and the keywords "Sporothrix AND sporotrichosis" resulted in a total of 395 publications for the period of 1975 to 2005, four decades. In contrast, if the same searching criteria was applied for the timeline 2005 to 2020 , the result was 638 publications. This means an improvement from an average of 9.9 papers per year from 1975 to 2005 , to an average of 25.5 papers per year from 2005 to 2020 . In the last decade (2010-2020), the average of publications jumped to 52.1 per year. Not less relevant to mention is the higher impact index and number of citations on Sporothrix and sporotrichosis papers, that can be noticed mainly in the last two decades. This BJM Special Issue intends to contribute not only for the

Leila M. Lopes-Bezerra

leila.lopes@bidiagnostics.com.br

1 BIDiagnostics, Technology and Innovation Center (CIETEC), São Paulo Univervity, São Paulo, Brazil

2 Departamento de Análises Clínicas e Toxicológicas, Faculdade de Ciências Farmacêuticas, Universidade de São Paulo, São Paulo, Brazil improvement of these numbers but, mostly, to bring new and important research data to address some challenges in human and animal sporotrichosis. We, hereby, will shortly resume these publications.

A relevant factor we need to point out is the change in the disease paradigm after the consolidation of the knowledge that sporotrichosis is not limited to humans and to Sporothrix schenckii infection. Nowadays, sporotrichosis is no longer related to this single etiological agent that has low pathogenicity and causes a benign subcutaneous mycosis. This means that the literature must be carefully revised on the light of new statements reported in this special issue. There are now, at least, two other epidemiologically relevant pathogenic species, Sporothrix brasiliensis and Sporothrix globosa, that do not exactly fit on the previous knowledge about $S$. schenckii infection.

Additionally, it is important to highlight that the diagnostic and treatment protocols should be revised, mainly for zoonotic sporotrichosis caused by S. brasiliensis. Sporotrichosis caused by this species has a high mortality rate for domestic cats (Felis catus domesticus) and cannot be considered just as a subcutaneous mycosis due to the fact that a systemic infection is very common in cats. This new pathogen had also changed the transmission paradigm. Previously, sporotrichosis was known as an occupational disease predominant in rural areas, the "rose gardener's disease." Nowadays, the zoonotic transmission in urban areas and in the peridomicile has gained clinical-epidemiological importance due to the increment in cat to human transmission. Furthermore, besides the emergence of $S$. brasiliensis with a high incidence in cats and humans, another emergent species, $S$. globosa, is the main causative agent of human sporotrichosis in China, reported in hundreds of human cases. To face these new challenges, the current ISHAM / WG18 coordination, Dr. Leila M Lopes Bezerra (Brazil) and Dr. Shanshan Li (China), had proposed and organized local committees of specialists in sporotrichosis caused by $S$. brasiliensis and $S$. globosa, in Brazil and China, respectively. These local groups of scientists are working as 
volunteers to discuss and try to understand if we are dealing with similar pathogens and/or similar pathologies. In this direction, several aspects need to be better discussed. This special issue highlights the recent progress in the study of this disease and its new etiological agents.

Do we need specific Guidelines for Human and Animal sporotrichosis? Trying to contribute to this answer the Brazilian committee on feline sporotrichosis is working since 2018 and generated a "Guideline for the management of feline sporotrichosis caused by S. brasiliensis" published in this special edition, Gremião et al. [1]. The goal is to alert professionals and health authorities for the challenges of the zoonotic disease caused by this new pathogen. It is a need to promptly diagnose and control this highly transmissible zoonotic infection that is no more limited to Brazil but, instead, is already reported in other South America countries.

The treatment of human and animal sporotrichosis is often performed with anti-fungal agents and the repertoire usually depends on several factors such as the clinical form of the disease, the longevity of the infection, the presence of other chronic illnesses in the patient, and the fungal species. However, in the last years, the emergence of anti-fungalresistant strains of Sporothrix species has been reported. Here Waller et al. [2, 3] discuss the emergence of Sporothrix species with in vitro anti-fungal resistance and the possible mechanisms for resistance. It has been demonstrated that the incubation temperature and growth phase may influence in vitro anti-fungal susceptibility profiles of $S$. schenckii and $S$. brasiliensis (sibling species of S. globosa). Song et al. [4], shows here that $S$. globosa sensitivity to antifungal compounds is dependent on the incubation temperature and the fungus growth phase. In recent years, different studies have demonstrated in vitro activity of some anti-inflammatory components. Borba-Santos et al. [5] discuss here the in vitro activity of ibuprofen, a nonsteroidal anti-inflammatory drug, against $S$. brasiliensis and $S$. schenckii.

The treatment of feline sporotrichosis is a challenge for veterinary clinicians since refractory cases may occur due to the patient immune response, to pharmacological management errors, and/or to anti-fungal resistance. Nakasu et al. [6] describe the therapeutic history of feline cases infected by itraconazole-resistant $S$. brasiliensis isolates in an endemic region of Southern Brazil. On the other hand, canine sporotrichosis is a poorly reported disease, and the majority of cases are from Rio de Janeiro, Brazil, as described here by Boechat et al. [7]. The authors report a $S$. schenckii infection in dogs, in the state of Rio de Janeiro, Brazil. To date, only S. brasiliensis infection has been described in dogs. Han and Kano [8] briefly discuss the status of feline sporotrichosis in Asia.

The effective diagnosis and treatment are critical to interrupt the chain of transmission of zoonotic sporotrichosis. Previously, the anti-SsCBF enzyme-linked immunosorbent assay (ELISA) test was shown to be useful as a diagnostic tool for human sporotrichosis. Baptista et al. [9] report here its applicability for feline sporotrichosis diagnosis and/or therapeutic follow-up. Grisolia et al. [10] present a seroepidemiological survey of the prevalence of sporotrichosis in four rural locations in the south of Minas Gerais state, Brazil.

Sporotrichosis in immunocompromised patients has a high morbidity and may cause deaths, particularly in patients with acquired immunodeficiency syndrome (AIDS) with low $\mathrm{T}$ CD4 counts. Here Cruz et al. [11] compared the genomic sequences of Sporothrix strains isolated from patients with confirmed sporotrichosis and a comorbidity, AIDS. The authors intend to detect changes in virulencerelated phenotypes and/or acquisition of anti-fungal resistance during the progression of these two conditions (sporotrichosis and HIV infection).

The innate immune response against Sporothrix spp. is important to control the infection. Cells such as macrophages play a key role on phagocytosis and presenting antigens to $\mathrm{T}$ cells. On the other hand, melanin is a virulence factor that can inhibit the innate immune functions of macrophages. Song et al. [12] demonstrate that $S$. globosa melanin can inhibit expression of antigen presentation-associated molecules during both the early and late stages of infection, proposing a new mechanism for this species to evade the host immunity in order to disseminate within the human host.

The efficiency of phagocytosis depends on several factors. The serum components may act as a bridge between the pathogens, inducing opsonization and increasing the phagocytosis. Guzman et al. [13] identified the serum proteins that bind to conidia of $S$. schenckii and test the ability of these proteins to induce phagocytosis.

The geographic distribution of Sporothrix spp. has a clear epidemiological impact. Toriello et al. [14] present a systematic review of sporotrichosis in Mexico that analyzes its epidemiology, geographic distribution, and diagnosis. Barreto et al. [15] showed here the ecological niche of the two circulating species of the Sporothrix genus found in Venezuela. Rudramurthy et al. [16] studied 63 cases of sporotrichosis of geographically diverse origin from India.

\section{References}

1. Gremião, I.D.F., Martins da Silva da Rocha, E., Montenegro, H. et al. Guideline for the management of feline sporotrichosis caused by Sporothrix brasiliensis and literature revision. Braz J Microbiol (2020). https://doi.org/10.1007/s42770-020-00365-3

2. Waller SB, Dalla Lana DF, Quatrin PM et al (2020) Antifungal resistance on Sporothrix species: an overview. Braz J Microbiol. https://doi.org/10.1007/s42770-020-00307-z

3. Waller SB, Ripoll MK, Madrid IM et al (2020) Susceptibility and resistance of Sporothrix brasiliensis to branded and compounded 
itraconazole formulations. Braz J Microbiol. https://doi.org/10. 1007/s42770-020-00280-7

4. Song Y, Li S, Shi Y et al (2020) In vitro antifungal susceptibility of Sporothrix globosa isolates from Jilin Province, northeastern China: comparison of yeast and mycelial phases. Braz J Microbiol. https://doi.org/10.1007/s42770-020-00316-y

5. Borba-Santos LP, Nucci M, Ferreira-Pereira A et al (2020) AntiSporothrix activity of ibuprofen combined with antifungal. Braz J Microbiol. https://doi.org/10.1007/s42770-020-00327-9

6. Nakasu CCT, Waller SB, Ripoll MK et al (2020) Feline sporotrichosis: a case series of itraconazole-resistant Sporothrix brasiliensis infection. Braz J Microbiol. https://doi.org/10.1007/ s42770-020-00290-5

7. Boechat JS, Pereira SA, de Sá Machado AC et al (2020) Canine sporotrichosis: polyphasic taxonomy and antifungal susceptibility profiles of Sporothrix species in an endemic area in Brazil. Braz J Microbiol. https://doi.org/10.1007/s42770-020-00328-8

8. Han HS, Kano R (2020) Feline sporotrichosis in Asia. Braz J Microbiol. https://doi.org/10.1007/s42770-020-00274-5

9. Baptista VS, Mothé GB, Santos GMP et al (2020) Promising application of the SsCBF ELISA test to monitor the therapeutic response of feline sporotrichosis caused by Sporothrix brasiliensisfrom Brazilian epidemics. Braz J Microbiol. https://doi.org/10. 1007/s42770-020-00362-6

10. Grisola JC, Santos LA, Coelho LML et al (2020) Seroepidemiological survey on sporotrichosis-infection in rural areas of the south of Minas Gerais state, Brazil. Braz $\mathrm{J}$ Microbiol. https://doi.org/10.1007/s42770-020-00279-0
11. Cruz ILR, Freitas DFS, de Macedo PM et al (2020) Evolution of virulence-related phenotypes of Sporothrix brasiliensis isolates from patients with chronic sporotrichosis and acquired immunodeficiency syndrome. Braz J Microbiol. https://doi.org/10.1007/ s42770-020-00297-y

12. Song Y, Yao L, Zhen Y, Cui Y, Zhong S, Liu Y, Li S (2020) Sporothrix globosa melanin inhibits antigen presentation by macrophages and enhances deep organ dissemination. Braz $\mathrm{J}$ Microbiol. https://doi.org/10.1007/s42770-020-00345-7

13. Guzman Beltrán S, Sanchez Morales J, González Canto A et al (2020) Human serum proteins bind to Sporothrix schenckii conidia with differential effects on phagocytosis. Braz J Microbiol. https:// doi.org/10.1007/s42770-020-00276-3

14. Toriello C, Brunner-Mendoza C, Ruiz-Baca E et al (2020) Sporotrichosis in Mexico. Braz J Microbiol. https://doi.org/10. 1007/s42770-020-00387-x

15. Barreto L, Velásquez G, Mendoza M et al (2020) Geographical distribution and ecological niche modeling of the etiological agents of human sporotrichosis in Venezuela. Braz J Microbiol. https://doi. org/10.1007/s42770-020-00306-0

16. Rudramurthy SM, Shankarnarayan SA, Hemashetter BM et al (2020) Phenotypic and molecular characterisation of Sporothrix globosa of diverse origin from India. Braz J Microbiol. https:// doi.org/10.1007/s42770-020-00346-6

Publisher's note Springer Nature remains neutral with regard to jurisdictional claims in published maps and institutional affiliations. 